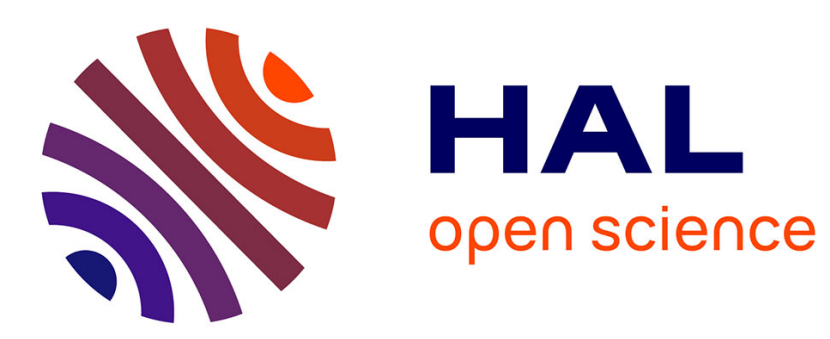

\title{
Game Theoretic Lane Change Strategy for Cooperative Vehicles under Perfect Information
}

Andres Ladino, Meng Wang

\section{To cite this version:}

Andres Ladino, Meng Wang. Game Theoretic Lane Change Strategy for Cooperative Vehicles under Perfect Information. Traffic Information and Control, Institution of Engineering and Technology, pp.147-162, 2020, 10.1049/PBTR026E_ch7 . hal-03227067

\section{HAL Id: hal-03227067 \\ https://hal.science/hal-03227067}

Submitted on 16 May 2021

HAL is a multi-disciplinary open access archive for the deposit and dissemination of scientific research documents, whether they are published or not. The documents may come from teaching and research institutions in France or abroad, or from public or private research centers.
L'archive ouverte pluridisciplinaire HAL, est destinée au dépôt et à la diffusion de documents scientifiques de niveau recherche, publiés ou non, émanant des établissements d'enseignement et de recherche français ou étrangers, des laboratoires publics ou privés. 


\title{
Chapter 7 \\ Game Theoretic Lane Change Strategy for Cooperative Vehicles under Perfect Information
}

\author{
Andres Ladino ${ }^{1}$ Meng Wang ${ }^{2}$
}

Lane change maneuvers are main causes of traffic turbulence at highway bottlenecks. We propose a dynamic game framework to derive the system optimum strategy for a network of cooperative vehicles interacting at a merging bottleneck. Cooperative vehicles on the highway mainline seek for optimal strategies (i.e. whether and when to perform courtesy lane change to facilitate the merging vehicle) to minimize their cost, while taking into account potential future interactions at the merging section while minimizing the distance travelled on the acceleration lane. An optimal strategy is found by minimizing the joint cost of all interacting vehicles while respecting behavioral and physical constraints. Numerical examples show the feasibility of the approach in capturing the nature of conflict and cooperation during the merging process and demonstrate the benefits of sharing information and cooperative control for connected automated vehicles.

\subsection{Introduction}

Social and economical development comes with an increased effect on traffic congestion and safety risks in terms of accidents. Planning, design, and deployment of such systems face new challenges everyday [1]. In particular, when multiple connected vehicles interact and exchange information, the problem of decision-making under conflicting situations with multiple vehciles as players emerges, especially at network discontinuities such as highway on-ramps [2]. In the context of traffic flow theory, these discontinuities are often bottlenecks with characterized highway capacity. In order to optimize the utility of the road network at merges, vehicular flow control has been proposed on the infrastructure side via ramp metering and variable speed limits strategies [3].

From cooperative systems' perspective, quite some advancements have been achieved in designing platoon or vehicle longitudinal controllers to stabilize platoon and traffic via Cooperative Adaptive Cruise Control (CACC) $[4,5,6]$. Nonetheless, it remains an open research question how to design the decision-making and (tra-

\footnotetext{
${ }^{1}$ University Gustave Eiffel, LICIT, F-69675 Lyon, France, andres.ladino@univ-eiffel.fr

${ }^{2}$ Delft University of Technology, 2628 CN, Delft, The Netherlands, m.wang@ tudelft.nl
} 
jectory) planning systems for lane change maneuvers of Connected \& Automated Vehicles (CAVs) such that collision is avoided, safety is guaranteed and traffic efficiency is maximized.

Several strategies were reported to deal with merging situation, most of which act on the longitudinal speed regulation. A set of scenarios was summarized in [4], describing vehicle interactions and active platoon policies at specific time instants. This finite state description did not formally address the theoretical properties of the solution. An optimal acceleration trajectory planing method for merging vehicles was proposed in [7], relying on a passing order decided by a higher decision layer. A specific trajectory design is proposed and fuzzy controllers are used as regulation strategies [8]. More recently, [9] proposed a bi-level control strategy to solve the merging process under mixed traffic conditions. However, it does not include the courtesy lane change of the mainline vehicles as the decision alternative. [10] formulated a stochastic switched system model to analyze how platoon-induced congestion varies with the fraction of platooned vehicles at merge, yet the decisions on when and where to split the platoon is not addressed. For a more complete literature review on this topic, we refer the reader to [11].

The merge situation can be modeled as a negotiation process between vehicles on the main carriageway and vehicles on the on-ramp willing to join the highway (See Fig. 7.1). A game theoretical framework was proposed in [12] where interacting CAVs predict and determine discrete desired lane sequences and continuous accelerations to minimize a cost function reflecting undesirable future situations. The computational load of this approach makes the real-time application a daunting task. A similar approach was considered in [13], where the constraints of the changing lane are formulated as a Mixed Logical Dynamical (MLD) model and the final control problem is cast via Mixed Integer Linear Programming (MILP). The framework assumes non-cooperative nature of automated vehicles. The majority of the aforementioned work did not consider the cooperative nature of interacting vehicles and some even needs another framework to put in the operational layer acceleration maneuvers. The lane change decisions are assumed to be selfish just for the benefit of a single agent, instead of cooperative to optimize the collective traffic performance.

This chapter puts forward a dynamic game framework to derive system optimum strategies for a network of cooperative vehicles interacting at a merging bottleneck. Cooperative vehicles traveling along the highway main lane seek to maximize an individual payoff by minimizing a running cost in a finite time horizon (i.e. whether and when to perform courtesy lane change to facilitate the merging vehicle). To minimize such cost, specific penalties are given for deviations from their desired driving conditions while taking into account the predicted action of merging vehicles. Merging vehicles minimize the distance travelled on the acceleration lane in addition to the same cost terms of the mainline vehicles, and predict the reaction of mainline vehicles responding to their merging decisions (i.e. whether and when to merge in the prediction horizon). An optimum strategy is found by minimizing the joint cost of interacting vehicles while respecting behavioral and physical constraints. Properties of the games and existence of solutions will be provided in this work. 
To solve the problem, a simplified discrete formulation of longitudinal vehicle dynamics is formulated. The longitudinal model is distributed, e.g. only interacting under predecessor-follower topology, and can be easily adapted to capture platooning systems dynamics. The full dynamic game is then cast as a set of sub-problems regularly expressed as standard optimal control problems that can be solved by mixedinteger quadratic/linear programming. Several examples at simulation level show the feasibility of the approach in capturing the nature of cooperation.

The operational assumptions and problem setup are explained with more detail in Section 7.2, then the model including longitudinal and lateral dynamics is explained in Section 7.3. The lane change decision action is cast as a dynamic game in Section 7.4. Numerical examples are shown in Section 7.5.

\section{Notation}

We denote in general single states with italic letters, $x_{i}$ hence denotes the value of the variable $x$ for a specific vehicle $i$, vectors collecting a set of variables are denoted as $\mathbf{x} \in \mathbb{R}^{|\mathscr{A}|}$. The symbol $\mathbb{R}^{|\mathscr{A}|}$ for a finite set $\mathscr{A}$ denotes the set of real vectors indexed by elements of $\mathscr{A},|\mathscr{A}|$ is the number of elements of the set $\mathscr{A}$. For $x \in \mathbb{R}^{n}, x>0$ is meant component-wise. Matrices are denoted with capital letters $A=\left[a_{i j}\right]$ where elements are denoted as $a_{i j}, \rho(A)$ denotes operation such as the spectral radius of the matrix The identity matrix of suitable dimensions is denoted $\square$.

\subsection{Problem Formulation}

In this paper we consider the situation shown in Fig. 7.1. Let $\mathscr{V}=\{1, \ldots, n\}$ be a group of CAV traveling along a road infrastructure composed by specific lanes labeled $\sigma=\{1,2,3\} \in \mathbb{N}$ from most right to most left. Let denote $\sigma_{i}(k)$ the lane occupied by vehicle $i$ at a specific instant of time $k$. Two vehicles $i, j$ traveling in different lanes $\sigma_{i} \neq \sigma_{j}$ are going to perform a merging negotiation at a current time $k_{0}$ in a time horizon of $N$ steps.

Two types of vehicle maneuvers can be conceived as possible in this situation. First, as shown in Fig. 7.1a the $i$-th vehicle in the platoon can modify its lateral position (in discrete lanes) to a new state $\sigma_{i}(k)=\sigma_{i}\left(k_{0}\right)+1$, while other vehicles in the platoon will keep the same position $\sigma_{i^{-}}(k)=\sigma_{i^{-}}\left(k_{0}\right) \forall i^{-} \in \mathscr{I} \backslash i$. In this case, a lateral decision operates over the vehicle $i$. A second situation can be envisaged as shown in Fig. 7.1b, the decision is taken at the level of the longitudinal control where a vehicle $i$ performs a maneuver to pass vehicle $j$ or yields in courtesy to open a gap where the $j$ vehicle will insert in front of vehicle $i$. Control maneuvers for this situation can be designed under knowledge of the state of the inserting vehicle $j$ [9]. In this case a longitudinal decision operates over vehicle $i$.

The decision-making and control system follows a hierarchical setting, where the decision-making module is placed on top of a motion control module [9]. This decision-making is based on a dynamic game framework [12]. It takes into account the current state information of the dynamic driving environment, which consists 


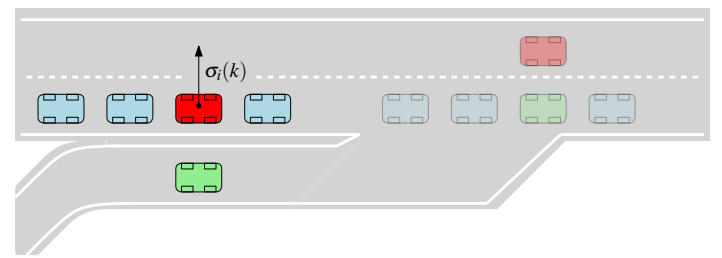

(a) Lateral control maneuver

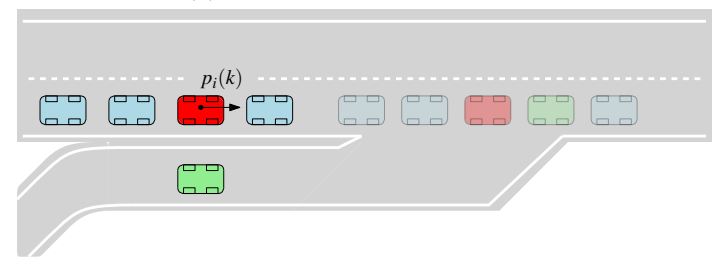

(b) Longitudinal control maneuver

Figure 7.1: Control actions for cooperative lane change maneuvers. In this case the red CAV illustrates two behaviors to open gaps for the inserting vehicle in green

of surrounding cooperative/non-cooperative vehicles. The state information can be estimated from measurements of on-board sensors or information transmitted via V2V communication devices. The interacting vehicles negotiate and jointly decide whether and when to change lane to optimize a joint cost/payoff function, taking into account the dynamic process as a response to the lane change actions. The lane change time is transferred to the lower level as the command to start lane change execution process. We focus on the tactical decisions while omitting the details in the vehicle lateral dynamics. The control problem can be cast as follows: Determine the lateral optimal control strategy such that a joint payoff/cost for vehicle $i$ and $j$ is maximized/minimized.

\subsection{Highway traffic system dynamics}

For the setting under consideration we consider two dimensions in the space of decision, longitudinal and lateral dynamics.

\subsubsection{Longitudinal dynamics}

The spacing with respect to the preceding vehicle and longitudinal position for vehicle $i$ is cast into discrete formulation as:

$$
\begin{aligned}
& s_{i}(k+1)=s_{i}(k)+\left(v_{l}(k)-v_{i}(k)\right) \Delta t \\
& p_{i}(k+1)=p_{i}(k)+v_{i}(k) \Delta t
\end{aligned}
$$

where $k \in \mathbb{Z}^{+}$denotes the discrete time index and $\Delta t$ is the time step size. The collection $\mathbf{p}, \mathbf{s}, \mathbf{v} \in \mathbb{R}^{n}$ denote vehicle's position, the headway space and the longitudinal speed respectively. It is convenient to define error terms to design control law. 
Hence, let

$$
\begin{array}{r}
e_{0, i}^{v}(k)=v_{0, i}-v_{i}(k) \\
e_{l, i}^{v}(k)=v_{l}(k)-v_{i}(k)
\end{array}
$$

where $v_{0, i}$ denotes the desired speed of vehicle $i$ and the subscript $l \in \mathscr{V} \cup\{j\}$ denotes the index of the direct leader of vehicle $i$. A feedback control law can be formulated as:

$$
v_{i}(k+1)=k_{0} e_{0, i}^{v}(k)+k_{l} e_{l, i}^{v}(k)
$$

$k_{0}, k_{l}$ are feedback gains for the errors to the desired speed and the predecessor speed respectively.

Safety conditions are guaranteed by imposing constraints on these dynamics. Hence, the vehicle dynamics are subject to the following linear constraints:

$$
\begin{array}{r}
a_{\min } \Delta t \leq v_{i}(k+1)-v_{i}(k) \leq a_{\max } \Delta t \\
v_{\min } \leq v_{i}(k) \leq v_{\max } \\
s_{i}(k) \geq v_{i}(k) t_{\min }+s_{0}
\end{array}
$$

where $t_{\min }$ denotes the minimum time gap between two vehicles on the same lane. $s_{0}$ denotes the minimum spacing between two vehicles. Constraint (7.7) states that any leader-follower space headway should keep some safe distance at any time instant $k$. $a_{\min }, a_{\max }, v_{\min }, v_{\max }$ represent boundaries in acceleration and speed correspondingly.

We remark that the simplified vehicle dynamic model with control input is flexible in terms of the error term definition. We choose the current form to capture the heterogeneous choice of desired speed by system users, while acknowledging that this is not the unique model for CAV platoons. If we use the gap error:

$$
\begin{gathered}
e_{i}^{s}(k+1)=s_{i}(k)-v_{i}(k) t_{d}-s_{0} \\
v_{i}(k+1)=k_{s} e_{i}^{s}(k)+k_{l} e_{l, i}^{v}(k)
\end{gathered}
$$

where $t_{d}$ denotes the desired time gap of ACC/CACC systems and $k_{s}$ denotes the feedback gain. The model can describe CACC platoon dynamics with proper tuning of feedback gains [14].

\subsubsection{Lateral dynamics}

We use the discrete lane change decision $\delta$ as the control decision variable, $\delta_{i} \in$ $\mathscr{D}:=\{-1,0,1\}$ where $\{-1,0,1\}:=\{$ change right, no lane change, change left $\}$. In the paper we assume only one lane change during the prediction horizon, but the framework is general to include multiple lane changes in the horizon [12]. This single switch aims to reduce the computational burden of the approach.

We use the travel lane of vehicle $i, \sigma_{i}(k)$ as the discrete state variable at time $k$. The dynamics of the lateral behavior are determined by:

$$
\sigma_{i}(k+1)=\sigma_{i}(k)+\delta_{i}(k)
$$

We assume lane change can take place as long as the gap is sufficiently large according to (7.7). We introduce the general possible maneuvers for all vehicles traveling 
along highways, nonetheless it is worth mentioning that the space of decision for the lateral maneuvers can be constrained for each vehicle depending on the trip lane or specific infrastructure policies.

\subsubsection{Lane change and dynamic communication topology}

The leader-follower pair is dynamic as a result of lane changes for the group of $n$ CAVs. Let a graph $\mathscr{G}=\{\mathscr{V}, \mathscr{E}\}, \mathscr{V}$ represents the set nodes consisting in all CAVs within the network and $\mathscr{E}=\{\mathscr{V} \times \mathscr{V}\}$ the set of edges representing a relationship between leaders and followers. Then $\mathscr{E}=\left\{\varepsilon_{i l}=1\right\}$ if vehicle $l$ is the leader of vehicle $i$ at specific sample time $k, 0$ otherwise. The adjacency matrix of $\mathscr{G}$ is concentrated in the squared matrix $A_{g}=\left[\varepsilon_{i j}\right]$. In general thanks to the lane change model (7.8), the set $\mathscr{E}$ is dynamic in time.

\subsubsection{Closed loop dynamics}

Let suppose a uniform formation where the desired speeds for all vehicles are the same and constant $v_{0, i}=\bar{v}_{0}$. For system (7.1), and combining with (7.4), it is possible to write the closed loop system as:

$$
\begin{aligned}
& s_{i}(k+1)=s_{i}(k)+\left(v_{l}(k)-v_{i}(k)\right) \Delta t \\
& v_{i}(k+1)=k_{0}\left(\bar{v}_{0}-v_{i}(k)\right)+k_{l}\left(v_{l}(k)-v_{i}(k)\right)
\end{aligned}
$$

Gathering all individual systems $i$ into an algebraic equation, it can be expressed as:

$$
\left[\begin{array}{l}
\mathbf{s}(k+1) \\
\mathbf{v}(k+1)
\end{array}\right]=\underbrace{\left(\begin{array}{cc}
\square & \left(A_{g}-\square\right) T \\
\mathbb{0} & K_{l}\left(A_{g}-\square\right)-K_{0}
\end{array}\right)}_{\bar{A}}\left(\begin{array}{l}
\mathbf{s}(k) \\
\mathbf{v}(k)
\end{array}\right)+\left(\begin{array}{c}
0 \\
K_{0} \overline{\mathbf{v}}_{0}
\end{array}\right)
$$

where $K_{0}, K_{l}, T$ are diagonal matrices in $\mathbb{R}^{n \times n}$ with corresponding elements $k_{0}, k_{l}, \Delta t$ in their diagonal. $\mathbb{0}, \mathbb{0}$ are the identity and the zero matrices of corresponding dimensions. $\overline{\mathbf{v}}_{0} \in \mathbb{R}^{n}$ is the constant vector containing on each element $\bar{v}_{0}$. $A_{g}$ is the adjacency matrix of the network topology (see 7.3.2).

It can be shown that if the spectral radius $\rho(\bar{A}) \leq 1, \rho(A):=\{\max |\lambda|: \lambda=$ $\operatorname{eig}(A)\}$ then the system (7.10) is stable.

\subsection{Game theoretic formulation of the lane change decision problem}

In this section we propose the dynamic game formulation for the lane change control maneuver.

\subsubsection{Dynamic lane change game formulation}

A vehicle traveling along a specific lane can establish a lateral decision denoted as a lane change strategy within a finite future time horizon $N$. 
Definition 1 (Lane change strategy). A vehicle lane change strategy from lane $\sigma_{\ell} \rightarrow$ $\sigma_{\ell^{+}}$is defined as the sequence:

$$
\begin{aligned}
& \xi_{\delta}=\left\{\sigma\left(k_{0}\right), \sigma\left(k_{0}+1\right), \ldots, \sigma\left(k_{0}+N-1\right)\right\} \\
& \sigma\left(k^{\star}\right)=\sigma_{\ell} \\
& \sigma\left(k^{\star}+1\right)=\sigma_{\ell^{+}} \\
& \sigma(k+1)=\sigma(k)+\delta(k), \\
& \sum_{k=0}^{N-1}|\delta(k)|=1
\end{aligned}
$$

$\xi_{\delta}$ represents the sequence associated to a particular lateral control $\delta(k)$ which induces the choice lane changing maneuver at $k^{\star}$ in the horizon $N$.

Consider the case of Fig. 7.2 where cooperative vehicles work together to find the best strategy that maximize the utility of the system as a whole. The objective of

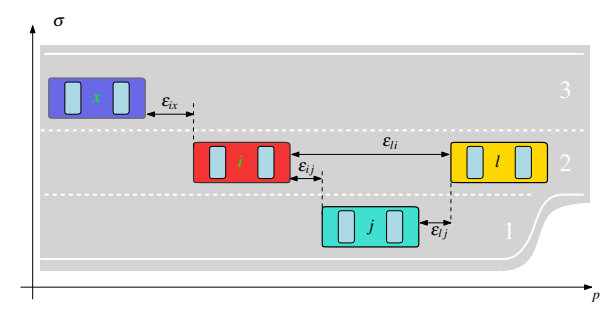

Figure 7.2: Lane change dynamic game. The controlled CAV in red optimizes the decision making between yielding at the merging time and changing lane.

the dynamic game is to create a decision block that considers the trade off between two possible cases. First, the situation in which in a finite time horizon the vehicle $i$ performs a lane change maneuver to create the necessary gap for insertion as depicted in Fig. 7.1a and a second situation where the vehicle $j$ should wait for the mainline vehicle to yield the necessary gap to so that the merging maneuver is performed without violating constraints. It is worth remarking that both vehicles $i, j$ will play a game in a finite horizon time that lead to a decision on lane changing for vehicle $j$. The cost for each vehicle is measured by undesirable situations:

$$
\begin{aligned}
L_{i}\left(\mathbf{p}(k), \mathbf{v}(k), \delta_{i}(k)\right)= & \beta_{1}\left|e_{0, i}^{v}(k)\right|+\beta_{2}\left|e_{l, i}^{v}(k)\right| \\
& +\beta_{3}\left|v_{i}(k+1)-v_{i}(k)\right| \\
& +\beta_{4}\left|\sigma_{i}(k)-\sigma_{i}^{*}\right| \\
& +\beta_{5}\left|\delta_{i}(k)\right| \\
& -\min \left\{0, \beta_{6}\left(p_{j}(k)-p_{j, \text { end }}\right)\right\}
\end{aligned}
$$

where $\beta_{g}, g \in\{1,2,3,4,5,6\}$ are the weights on different cost terms. $p_{j, \text { end }}$ denotes the position of the end of a mandatory lane change section for vehicle $j$. The running cost function can be interpreted as follows:

- The first term encourages the vehicle to travel at its desired speed; 
- The second term encourages consensus on speed for each leader-follower pair;

- The third term favors smooth speed change and hence discourage sharp acceleration and deceleration;

- The fourth term penalizes deviation from desired lane $\sigma_{i}^{*}$ and the fifth term penalizes lane changes.

- The last terms penalizes potential failure for mandatory lane change. It favours early mandatory lane changes and increases when the distance to the end of the merging lane $p_{\text {end }}$ is decreasing.

The optimal control problem can be cast as an optimization of the running cost $L_{i}$ for each one of the players while other players have already decided. A dynamic game can be integrated within an optimal control problem where each one of the players fixes a specific strategy in particular for the lane change by targeting the specific value $\sigma_{i}^{*}$. Notice that each player $i$ has a finite number of strategies to choose by selecting specific $\delta_{i}$. In particular, when playing the game in between vehicle $i$ and vehicle $j$ it is possible to write the following finite horizon problem:

$$
\begin{array}{ll}
\min _{\delta_{i}(\cdot) \in \mathscr{D}} & \left.\sum_{g=i, j} \sum_{k=0}^{N-1} L_{g}\left(\mathbf{p}(k), \delta_{g}(k)\right)+\Phi_{g}(\mathbf{p}(N)), \delta(N)\right) \\
\text { s.t } & (7.1),(7.5),(7.7),(7.6),(7.8) \\
& \delta_{i}(k) \in \underline{D}=\{0,1\}, \text { only allow left lane changes } \\
& \sum_{k=0}^{N-1} \delta_{i}(k) \leq 1, \text { only allow one lane change }
\end{array}
$$

The objective of the former optimal control problem is to promote the minimization of the individual costs. This is formulated as an optimization problem, where one seeks the optimal lane change decision trajectories for each vehicle $i$ in a prediction horizon $N$ to maximize the payoff function of the whole group. In fact each one of the player should maximize a payoff given by:

$$
J_{i}\left(\mathbf{p}(k), \mathbf{v}(k), \delta_{i}(k)\right)=-\sum_{k=0}^{N-1} L_{i}\left(\mathbf{p}(k), \mathbf{v}(k), \delta_{i}(k)\right)
$$

The dynamic game entails prediction of the payoff over a time horizon with $N$ steps: $[0, N]$. We consider $N$ to be sufficiently large and therefore set the terminal cost $\Phi=0$. The player $i$ will select a strategy among a finite set $\mathscr{D}$ of strategies.

\subsubsection{Existence of equilibrium}

We first introduce general conditions for the existence of equilibrium in a two player game setup. Let consider the vehicle $i$ and all the possible set of finite strategies $\mathscr{A}=\left\{a_{1}, a_{2}, \ldots, a_{r}\right\}$ to be chosen for the lateral decisions. Let $\mathscr{B}=\left\{b_{1}, b_{2}, \ldots, b_{q}\right\}$ the possible decisions for the $j$ vehicle traveling in the on-ramp lane. It is worth to remark that a vehicle $i, j$ have at most $\left|\mathscr{A}^{i}\right|=\left|\mathscr{B}^{i}\right|=2^{N-1}$ possibilities to change lane during a future finite horizon. 
Theorem 1 (Existence of Nash Equilibrium [15]). Assume that the sets of strategies $\mathscr{A}, \mathscr{B}$ are compact, convex subsets of $\mathbb{R}^{n}$. Let the payoff functions $\phi^{\mathscr{A}}, \phi^{\mathscr{B}}$ be continuous. If $a \mapsto \phi^{\mathscr{A}}(a, b)$ results in a concave function of $a, \forall b \in \mathscr{B}$ and $b \mapsto \phi^{\mathscr{B}}(a, b)$ is a concave function of $b, \forall a \in \mathscr{A}$ then the game is called non-cooperative and it admits a Nash equilibrium.

Proof. To proof the existence of the Nash equilibrium, it is important to proof the compactness and convexity of the best replies. The existence of the equilibrium is then supported by Kakutani's fixed point theorem, that shows that each best response correspondence has a fixed point. Consider the best response maps $r^{\mathscr{A}}(b), r^{\mathscr{B}}(a)$ defined as:

$$
\begin{aligned}
& r^{\mathscr{A}}(b)=\left\{a \in \mathscr{A}, \phi^{\mathscr{A}}(a, b)=\max _{\theta} \phi^{\mathscr{A}}(\theta, b)\right\} \\
& r^{\mathscr{B}}(a)=\left\{b \in \mathscr{B}, \phi^{\mathscr{B}}(a, b)=\max _{\theta} \phi^{\mathscr{A}}(a, \theta)\right\}
\end{aligned}
$$

The continuity of $\phi^{\mathscr{B}}$ causes the map $\phi^{\mathscr{B}}(a, b)$ to be continuous and establishes a closed set $\mathscr{G}(b)=\left\{(a, b) \mid b \in r^{\mathscr{B}}(a)\right\}$ where $\mathscr{G}(b) \subset \mathscr{B}$, correspondingly $\mathscr{G}(a) \subset \mathscr{A}$.

Since $\mathscr{B}$ is convex $b_{1} \theta+(1-\theta) b_{2}$ is and admissible convex combination for $\mathscr{B}$. In this case $\theta b_{1}+(1-\theta) b_{2} \in r^{\mathscr{B}}(a)$ since $\phi^{\mathscr{B}}\left(a, \theta b_{1}+(1-\theta) b_{2}\right) \geq \theta \phi^{\mathscr{B}}\left(a, b_{1}\right)+$ $(1-\theta) \phi^{\mathscr{B}}\left(a, b_{2}\right)$ due to the concavity property of $\phi^{\mathscr{B}}$. The same analysis can be conducted for $\phi^{\mathscr{A}}(b)$. This result leads to the fact that any combination of responses $(a, b)$ in fact belongs to the map of combinations $m(a, b) \mapsto r^{\mathscr{A}}(b) \times r^{\mathscr{B}}(a)$. Given that the sets $\mathscr{A}, \mathscr{B}$ are non empty and convex the map $m(a, b)$ is not empty. Then a fixed value can be found according to Kakutani's theorem.

Lemma 1 (Existence of Nash Equilibrium at fixed time). If $\sigma_{i}(k) \in \mathscr{D}$ then set of strategies A,B for two players admit a Nash equilibrium at time $k$.

Proof. Given that $|\sigma|=3$ and according to 1 , the number of strategies is countable. By extension of 1 it is straight forward to admit a Nash equilibrium at sample time $k$.

Definition 2 (Payoff function). Let be $J_{i}^{A}\left(\mathbf{p}(k), \mathbf{v}(k), a_{\delta}, b_{\delta}\right)$ the function defining the payoff after a player decides among the set of strategies $A$ as:

$$
\begin{aligned}
J_{i}^{A}\left(\mathbf{p}(k), \mathbf{v}(k), a_{\delta}, b_{\delta}\right)= & \psi_{i}(p(N))- \\
& \sum_{k=0}^{N-1} L_{i}\left(\mathbf{p}(k), a_{\delta}, b_{\delta}\right)
\end{aligned}
$$

In Definition $2, L_{i}$ is defined as the running cost while the $\psi_{i}$ is called the final cost.

Assumption 1 (Available game information). Dynamic (7.1) is well known for each one of the participant of the games.

The same as $J_{i}^{A}\left(\mathbf{p}(k), \mathbf{v}(k), a_{\delta}, b_{\delta}\right), J_{j}^{B}\left(\mathbf{p}(k), \mathbf{v}(k), a_{\delta}, b_{\delta}\right)$ and the sample time $k$ is considered synchronous in vehicles $i, j$. 


\subsubsection{Properties of the lane change dynamic game}

Consider the full dynamics expressed in equation (7.10) jointly with (7.8) and enclosed in the form $\mathbf{x}(k+1)=f(\mathbf{x}(k), \delta(k))=A \mathbf{x}(k)+M \delta(k) . \mathbf{x}^{T}=\left(\begin{array}{lll}\mathbf{p}^{T} & \mathbf{v}^{T} & \sigma^{T}\end{array}\right)$. In a particular case where two players are defining a game it is possible to define split dynamics and running costs as:

$$
\begin{aligned}
\mathbf{x}(k+1) & =A \mathbf{x}(k)+M_{1} \delta_{1}(k)+M_{2} \delta_{2}(k) \\
L_{i}(k) & =L_{i 1}\left(\mathbf{x}(k), \delta_{1}(k)\right)+L_{i 2}\left(\mathbf{x}(k), \delta_{2}(k)\right)
\end{aligned}
$$

Remark 1 (Finding equilibrium via PMP). Let consider the system (7.17) with associated running cost (7.17). Let $\mathbf{x}^{*}(\cdot), \delta_{1}^{*}(\cdot), \delta_{2}^{*}(\cdot)$ be respectively the trajectory and open loop controls of two players in a Nash equilibrium. By definition this two controls provide corresponding solutions to the associated optimal control problems for each player. Applying the Pontryagin Maximum Principle the following are necessary conditions for the Nash equilibrium [16].

$$
\begin{aligned}
\mathbf{x}(k+1) & =\mathbf{x}(k)+M_{1} \delta_{1}^{\natural}(k)+M_{2} \delta_{2}^{\natural}(k) \\
\lambda_{1}(k) & =\bar{A} \lambda_{1}(k+1)+\nabla_{\mathbf{x}} L_{11}\left(\mathbf{x}(k), \delta_{1}^{\natural}(k)\right) \\
\lambda_{2}(k) & =\bar{A} \lambda_{2}(k+1)+\nabla_{\mathbf{x}} L_{22}\left(\mathbf{x}(k), \delta_{2}^{\natural}(k)\right)
\end{aligned}
$$

where

$$
\begin{aligned}
& \delta_{1}^{\natural}=\underset{\delta_{1} \in \mathscr{D}}{\arg \max } \lambda_{1} M_{1} \omega-L_{11}\left(t, \mathbf{x}, \delta_{1}\right) \\
& \delta_{2}^{\natural}=\underset{\delta_{2} \in \mathscr{D}}{\arg \max } \lambda_{2} M_{2} \omega-L_{22}\left(t, \mathbf{x}, \delta_{2}\right)
\end{aligned}
$$

In order to solve the find the conditions for Nash equilibrium let define the Hamiltonian for the control problem (7.13) based on (7.17),(7.17):

$$
\begin{aligned}
H\left(\mathbf{x}(k), \delta_{1}(k), \delta_{2}(k)\right) & =\sum_{i \in \mathscr{I}}\left(L_{i 1}\left(\mathbf{x}(k), \delta_{1}\right)\right. \\
& \left.L_{i 2}\left(x(k), \delta_{2}(k)\right)\right)- \\
& \lambda_{i}\left(\mathbf{x}(k)+M_{1} \delta_{1}(k)+M_{2} \delta_{2}(k)\right)
\end{aligned}
$$

By considering the costate condition for $\lambda \in \mathbb{R}^{n}$ from the Pontryagin Maximum Principle [16]:

$$
\lambda_{i}(k)=\frac{\partial H}{\partial x_{i}}=\left(\frac{\partial H}{\partial x_{i}}\right)^{T} \lambda_{i}(k+1)+\frac{\partial L(k)}{\partial x_{i}}
$$

with the final condition $x(T)=0$, then it is possible to obtain the conditions in (7.18),(7.18). The optimal condition is derived from the fact that for a fixed lateral control $\bar{\delta}_{2}(\cdot)$, the optimal $\delta_{1}(\cdot)$ can be found via

$$
\delta_{1}^{*}(\cdot)=\underset{\delta_{1} \in \mathscr{D}}{\arg \min } H\left(\mathbf{x}(k), \delta_{1}(k), \bar{\delta}_{2}(k)\right)
$$


which can be transformed into a maximization problem where the player is maximizing the payoff function similar to (7.16), leading to

$$
\delta_{1}^{*}(\cdot)=\underset{\delta_{1} \in \mathscr{D}}{\arg \max }-H\left(\mathbf{x}(k), \delta_{1}(k), \bar{\delta}_{2}(k)\right)
$$

The stationary condition is necessary for optimality then by introducing (7.20) in to (7.23), we obtain:

$$
\begin{gathered}
0=-\frac{\partial L_{11}\left(\mathbf{x}(k), \delta_{1}(k)\right)+L_{12}\left(\mathbf{x}(k), \bar{\delta}_{2}(k)\right)}{\partial \delta_{1}}+ \\
\lambda_{1} \frac{\partial\left(x(k)+M_{1} \delta_{1}(k)+M_{2} \bar{\delta}_{2}(k)\right)}{\partial \delta_{1}}
\end{gathered}
$$

leading to (7.19a). In the same way (7.19b) can be obtained when the first player fixes its own strategy to a value $\bar{\delta}_{1}=\delta_{1}^{*}$. The nash equilibrium is obtained when the payoff for player 1 is maximized 7.23 with the best response of player 2 and viceversa [15]. In other words, no player can increase his payoff by single-mindedly changing his strategy, as long as the other player sticks to the equilibrium strategy.

In general the game here presented is a non-zero sum game, and as players in fact cooperate towards the common objective, given by the successful lane change. On the other hand, the scalability of this approach may suffer with long time horizons. In this case we propose an heuristic way to solve this algorithm [17].

\subsection{Numerical examples}

\subsubsection{Experimental setting}

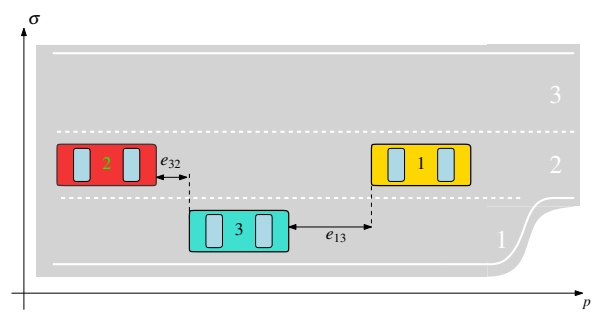

Figure 7.3: Scenarios

To test the working of the dynamic game framework, we conducted numerical examples. The scenario is set up as in Fig. 7.3. We simulate 3 vehicles, with Vehicle 2 and Vehicle 3 interacting with each other in the merging section. The initial conditions are: $p_{1}(0)=0 m, p_{2}(0)=-50 m v_{l 1}(0)=v_{l 2}(0)=v_{1}(1)=v_{2}(0)=30 \mathrm{~m} / \mathrm{s}$, $\sigma_{1}(0)=\sigma_{2}(0)=2, \sigma_{3}(0)=1, \sigma_{2}^{*}=\sigma_{3}^{*}=2$ (The desired lanes for both Vehicle 2 and Vehicle 3 are Lane 2, the right lane on the main freeway). $v_{3}(1)=25 \mathrm{~m} / \mathrm{s}$, $p_{3}(1)=-45 \mathrm{~m}$ for Scenario 1 and $p_{3}(1)=-35 \mathrm{~m}$ for Scenario 2. $v_{0}=30 \mathrm{~m} / \mathrm{s}, v_{\min }=$ $0 \mathrm{~m} / \mathrm{s}, v_{\max }=35 \mathrm{~m} / \mathrm{s}, a_{\min }=-5 \mathrm{~m} / \mathrm{s}^{2}, a_{\max }=2 \mathrm{~m} / \mathrm{s}^{2} \beta_{1}=0.2, \beta_{2}=0.2, \beta_{3}=0.5, \beta_{4}=$ $5, \beta_{5}=5, \beta_{6}=0.05, t_{\min }=0.5 s$. 


\subsubsection{Scenario 1: delayed merge}
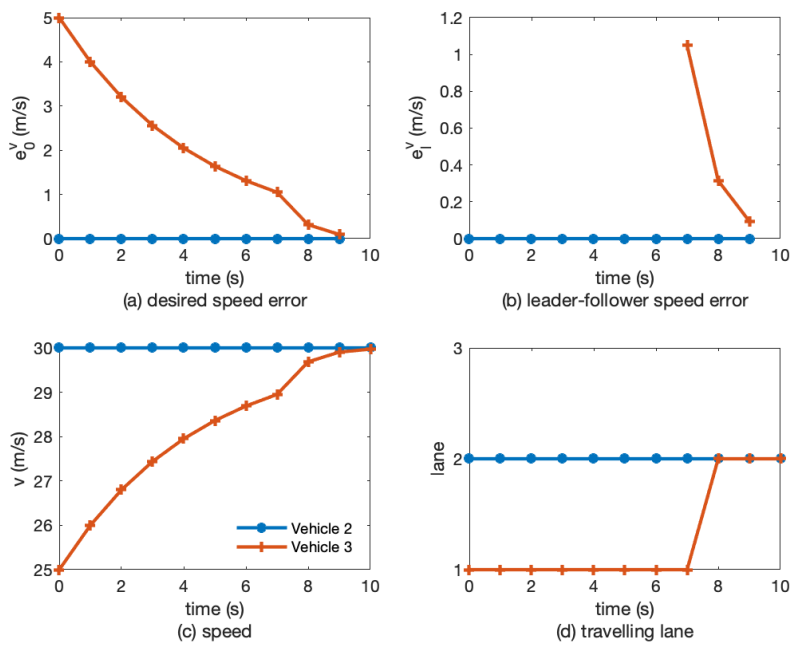

Figure 7.4: Delayed merge

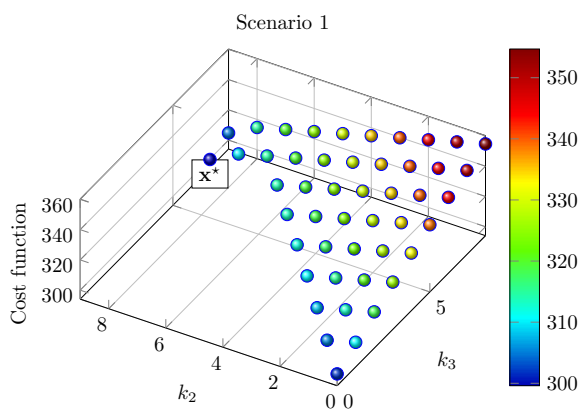

Figure 7.5: Overall cost for Scenario 1. $k_{i}$ represents the time to change lane of vehicle $i$

In Scenario 1, Vehicle 3 is 5 meters in front of Vehicle 2 but with a slower speed. The resulting cost of all vehicles and the cost of Vehicle 3 and Vehicle 2 are shown in Fig. 7.5. The best situation for Vehicle 3 is that Vehicle 2 performs courtesy lane change from Lane 2 to Lane 3, so that Vehicle 3 has a conflict-free merge. In this case, Vehicle 3 can change lane immediately to minimize its own cost. However, this leads to deviation of from the desired lane of Vehicle 2, leading to a cost of 50. The overall cost is not the optimum for the whole vehicle group. 
From the collective system perspective, the best strategy is that Vehicle 2 stays in the same lane and passes vehicle 3. Vehicle 3 waits for Vehicle 2 to pass until sufficient safety gap is developed in front, and changes lane at $k=7$ second.

Interestingly, if following a first-in-first-out strategy that is widely used in cooperative merging systems [11], it leads to the feasible strategy that is best for vehicle 3 , but not the best for the collective vehicle group.

Fig. 7.4 shows the system optimal solution, where the error on desired speed $e_{0}^{v}$, speed error to predecessor $e_{l}^{v}$, vehicle speed and lane sequence are depicted. Note that the change of increasing rate in speed for Vehicle 3 is due to fact that before the lane change, Vehicle 3 has no leader and it only accelerates towards the desired speed. When it changes lane, the both the error on desired speed and speed error to predecessor demand it to accelerate, resulting in an increase in speed change rate.

\subsubsection{Scenario 2: Courtesy lane change}

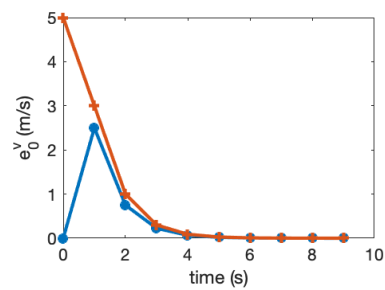

(a)

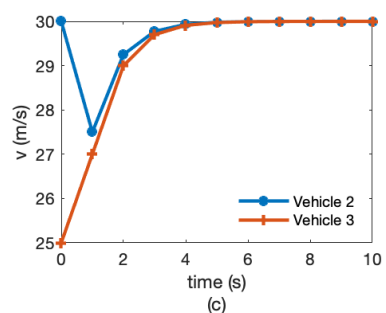

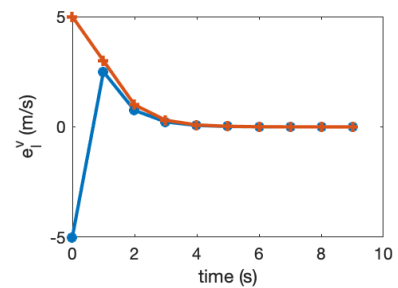

(b)

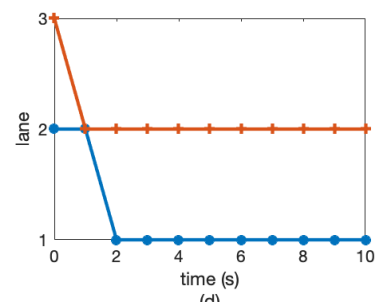

(d)

Figure 7.6: Courtesy lane change

In Scenario 2, Vehicle 3 is 15 meters in front of Vehicle 2. The resulting cost of all vehicles and the cost of vehicle 2 and 3 are shown in Fig. 7.7. In this case, it is not safe for Vehicle 3 anymore if Vehicle 2 still tries to pass Vehicle 3. Instead, the best strategy from the whole vehicle network perspective is that Vehicle 2 changes to left immediately and Vehicle 3 changes left immediately. This will minimize the lane preference cost and the cost of reaching the end of the merging section for Vehicle 3, at the expenses of lane preference cost for Vehicle 2. Fig. 7.6 shows the system optimal solution. This illustrates the very nature of cooperation: individual agents may have to compromise their own benefits for the best performance of the whole system. 


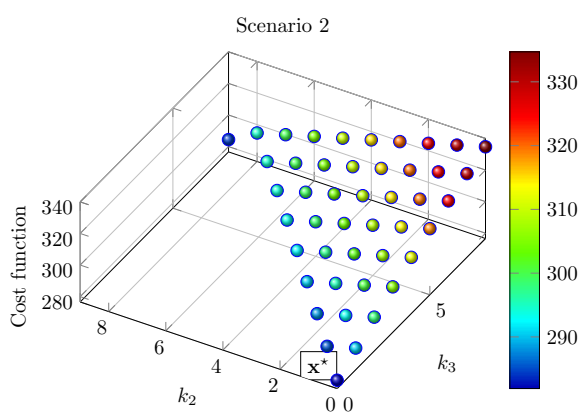

Figure 7.7: Overall cost for Scenario 2. Blank areas are infeasible strategies, i.e. due to violation of safety constraints

\subsection{Conclusion}

In this chapter, we proposed a dynamic game formulation for cooperative lane change maneuvers of automated vehicles at highway merges. Simplified vehicle longitudinal and lateral dynamics models are used to predict the system process under different lane change strategies. The framework captures the competitive and cooperative nature of the interactions between the merging vehicle and the mainline vehicle, and renders the design tractable to a range of mathematical tools related to optimal control and integer programming. The discrete dynamic model with control input substantially reduces the computational load for the dynamic merging game compared to previous work. Numerical examples demonstrate the potential of the approach in generating system optimum strategies as opposed to existing non-cooperative merging algorithms and the benefits of sharing information and joint optimization under a common goal.

Future research is directed to the scalability analysis of the proposed framework and efficient solution algorithms to a large network of cooperative vehicles and the assessment of effects of this framework on traffic operations.

\section{References}

[1] Hanappe F, Hudson A, Pelloux P, et al. Impacts and potential benefits of autonomous vehicles: From an international context to Grand Paris. Apur; 2018.

[2] Rios-Torres J, Malikopoulos AA. Automated and Cooperative Vehicle Merging at Highway On-Ramps. IEEE Transactions on Intelligent Transportation Systems. 2017;18(4):780-789.

[3] Papageorgiou M, Kiakaki C, Dinopoulou V, et al. Review of road traffic control strategies. Proceedings of the IEEE. 2003 12;91(12):2043-2067.

[4] Nowakowski C, Thompson D, Shladover SE, et al. Operational Concepts for Truck Maneuvers with Cooperative Adaptive Cruise Control. Transporta- 
tion Research Record: Journal of the Transportation Research Board. 2016 1;2559:57-64.

[5] Zhang Y, Wang M, Hu J, et al. Semi-Constant Spacing Policy for LeaderPredecessor-Follower Platoon Control via Delayed Measurements Synchronization. In: 21st IFAC World Congress; 2020. .

[6] Zhang Y, Bai Y, Hu J, et al. Control Design, Stability Analysis, and Traffic Flow Implications for Cooperative Adaptive Cruise Control Systems with Compensation of Communication Delay. Transportation Research Record. 2020;.

[7] Ntousakis IA, Nikolos IK, Papageorgiou M. Optimal vehicle trajectory planning in the context of cooperative merging on highways. Transportation Research Part C: Emerging Technologies. 2016 10;71:464-488.

[8] Milanés V, Godoy J, Villagra J, et al. Automated On-Ramp Merging System for Congested Traffic Situations. IEEE Transactions on Intelligent Transportation Systems. 2011 6;12(2):500-508.

[9] Duret A, Wang M, Ladino A. A hierarchical approach for splitting truck platoons near network discontinuities. Transportation Research Part B: Methodological. 2019 4;

[10] Jin L, Čičič M, Amin S, et al. Modeling the Impact of Vehicle Platooning on Highway Congestion. In: Proceedings of the 21st International Conference on Hybrid Systems: Computation and Control (part of CPS Week) - HSCC '18. ACM Press; 2018. p. 237-246.

[11] Rios-Torres J, Malikopoulos AA. A Survey on the Coordination of Connected and Automated Vehicles at Intersections and Merging at Highway On-Ramps. IEEE Transactions on Intelligent Transportation Systems. 2017;18(5):10661077.

[12] Wang M, Hoogendoorn SP, Daamen W, et al. Game theoretic approach for predictive lane-changing and car-following control. Transportation Research Part C: Emerging Technologies. 2015;58:73-92.

[13] Fabiani F, Grammatico S. A Mixed-Logical-Dynamical model for Automated Driving on highways. In: 2018 IEEE Conference on Decision and Control (CDC). IEEE; 2018. p. 1011-1015.

[14] Xiao L, Wang M, Schakel W, et al. Unravelling effects of cooperative adaptive cruise control deactivation on traffic flow characteristics at merging bottlenecks. Transportation Research Part C: Emerging Technologies. 2018;96:380 - 397.

[15] Bressan A. Non Cooperative Differential Games. A Tutorial. Penn State University; 2010.

[16] Lewis FL, Vrabie D, Vassilis LS. Optimal Control. 3rd ed. John Wiley \& Sons; 2012.

[17] Ladino A, Wang M. A Dynamic Game Formulation for Cooperative Lane Change Strategies at Highway Merges. In: 21st IFAC World Congress; 2020. 